\title{
NEPTUNE'S DEEP ATMOSPHERE REVEALED
}

\author{
Paul N. Romani
}

Science Systems and Applications, Inc.

Imke de Pater

Astronomy Department, University of California, Berkeley

Sushil K. Atreya

Department of Atmospheric, Oceanic and Space Sciences, The University of Michigan

\begin{abstract}
The brightness temperature of Uranus at 20 $\mathrm{cm}$ is $260 \pm 10 \mathrm{~K}$, while for Neptume it is $318 \pm 16 \mathrm{~K}$. Since $\mathrm{NH}_{3}$ is the dominant absorber at this wavelength we have modeled the microwave spectra of Neptune based upon an assumed deep gaseous mixing ratio of $\mathrm{NH}_{3}$ and subsequent loss into clouds. The difference between the two brightness temperatures implies that the $\mathrm{NH}_{3}$ mixing ratio below the level of cloud formation on Neptune compared to Uranus is lower by nearly 2 orders of magnitude. An alternative explanation is that the $20 \mathrm{~cm}$ radiation from Neptune is a combination of thermal plus synchrotron emission as proposed by de Pater and Goertz [1989].
\end{abstract}

\section{Introduction}

Since Neptune and Uranus both formed in the outer part of the solar nebula and are of similar masses, it is plausible to assume that they formed out of the same bulk material and underwent similar evolutions. There are observations that support this hypothesis. Analysis of the infrared spectrum of both planets yield similar $\mathrm{CH}_{4} \mathrm{mix}-$ ing ratios of approximately $2 \%$ below the $\mathrm{CH}_{4}$ ice cloud (at $\approx 80 \mathrm{~K}$ and 1.3 bars) and approximately the same temperature pressure structure in the 1 bar region [Orton et al., 1987]. Observations at radio wavelengths probe deeper into the atmospheres than the IR (up to 50-100 bars) with most of the opacity at centimeter wavelengths due to ammonia gas. At $3-6 \mathrm{~cm}$ ( $\mathrm{P} \lesssim 20$ bar ) both planets show a depletion of ammonia gas [de Pater and Massie, 1985]. Recently, the thermal radio spectrum of Uranus has been shown to be consistent with $\mathrm{NH}_{3}$ enriched by $>1-10$ times above solar and $\mathrm{H}_{2} \mathrm{~S}$ enriched by a few hundred times above solar at $\mathrm{P} \gtrsim 50-100$ bars; the $\mathrm{NH}_{3}$-gas mixing ratio is reduced at lower pressures in the atmosphere first by solution into a water cloud then by the formation of an $\mathrm{NH}_{4} \mathrm{SH}$ cloud [de Pater et al., 1989].

There are, however, differences between the two planets. Neptune has an internal heat source in excess of $100 \%$ of the absorbed solar energy, while the internal heat source on Uranus is less than $13 \%$ of the absorbed solar [Conrath et al., 1988]. In the stratosphere of Neptune methane is supersaturated relative to the cold trap

Copyright 1989 by the American Geophysical Union.

Paper number $89 \mathrm{GL} 01355$.

$0094-8276 / 89 / 89 \mathrm{GL}-01355 \$ 03.00$ (tropopause) mixing ratio but on Uranus methane in the stratosphere is cold trap limited [Orton et al., 1987]. Uranus' radio spectrum is relatively flat at $\sim 260 \mathrm{~K}$ between 6 and $20 \mathrm{~cm}$ but Neptune's brightness temperature increases from $\sim 200 \mathrm{~K}$ at $6 \mathrm{~cm}$ to $\sim 318 \mathrm{~K}$ at $20 \mathrm{~cm}$ [de Pater et al., 1989; de Pater and Richmond, 1989].

In this paper we apply to Neptune's radio spectrum the models we developed to study Uranus's [de Pater et al., 1989]. This work is the first attempt ever to use a cloud model to produce an $\mathrm{NH}_{3}$ distribution in Neptune's atmosphere, compute the resultant synthetic radio spectrum, and compare it to observations. Recently de Pater and Goertz [1989] proposed that the higher brightness temperature at $20 \mathrm{~cm}$ on Neptune compared to Uranus was due to synchrotron emission on Neptune adding to a thermal flux that was the same for Uranus. The primary question we address here is: Can we model the radio spectrum of Neptune with an $\mathrm{NH}_{3}$ mixing ratio in the deep atmosphere which is equal to or larger than the solar value, such that we match the $20 \mathrm{~cm}$ brightness temperature as well as the temperature at shorter wavelengths, i.e. an $\mathrm{NH}_{3}$ distribution similar to Uranus.

\section{Models}

\section{Atmospheric Model}

The cloud model used in this study is a simple lifting parcel model that has been described extensively before [de Pater et al., 1989]. The calculations start deep in the atmosphere below the formation of suspected clouds: aqueous ammonia solution, water ice, ammonia ice, hydrogen sulfide ice, ammonium hydrosulfide solid, methane ice, and argon ice, with a specified composition, temperature, and pressure. The model steps up in altitude and the new temperature is calculated from the dry adiabatic lapse rate and the new pressure by assuming hydrostatic equilibrium. The partial pressures of all constituents are calculated assuming the atmosphere to be an ideal gas. From the new temperature and empirical formulas the equilibrium saturation vapor pressures of all possible condensates are calculated. If the partial pressure of a condensate in the atmosphere exceeds its respective saturation vapor pressure, then a cloud forms and the atmospheric partial pressure of the condensing species is set equal to its equilibrium saturation pressure. Since the $\mathrm{NH}_{4} \mathrm{SH}$ cloud forms as a result of a reaction between $\mathrm{NH}_{3}$ and $\mathrm{H}_{2} \mathrm{~S}$, the test for cloud formation is that the equilib- 


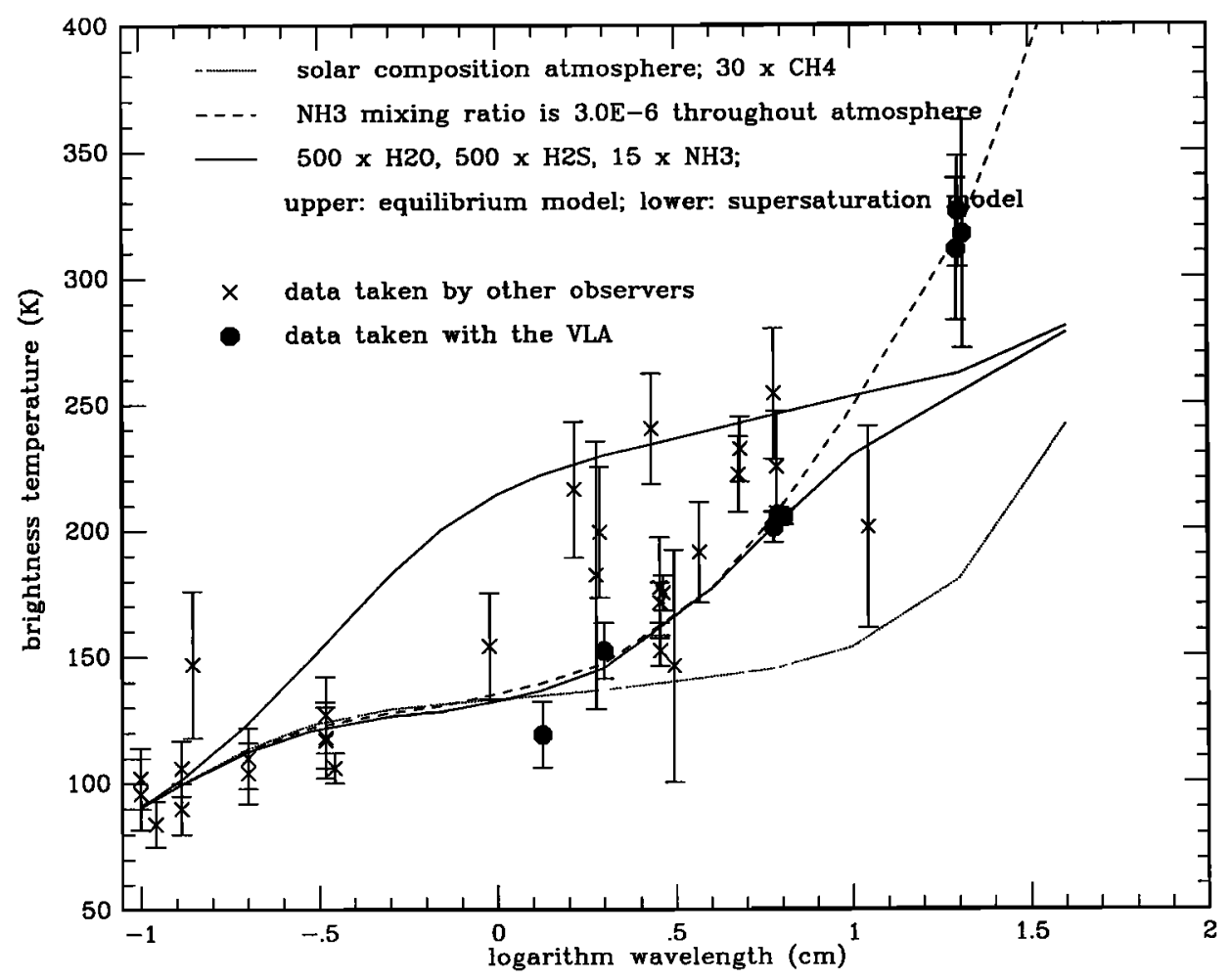

Fig. 1. Neptune's radio spectrum (from de Pater and Richmond) with superimposed model atmosphere calculations for assumed base level enrichments of $\mathrm{H}_{2} \mathrm{O}, \mathrm{NH}_{3}$, and $\mathrm{H}_{2} \mathrm{~S}$ relative to solar, as indicated on the figure. See text for further explanations.

rium constant is exceeded. And if the $\mathrm{NH}_{4} \mathrm{SH}$ cloud forms the $\mathrm{NH}_{3}$ and $\mathrm{H}_{2} \mathrm{~S}$ mixing ratios are reduced in equal molar quantities so the product of their atmospheric pressures now equals the equilibrium constant. If the aqueous ammonia cloud forms $\mathrm{H}_{2} \mathrm{~S}$ is dissolved into it. The model then steps up in altitude again using now either the dry or the appropriate wet adiabat. As the trace gases are removed from the atmosphere by condensation, "dry" air (an $\mathrm{H}_{2}$-He mixture) is entrained into the parcel to ensure the volume mixing ratios sum to one. This cycle is repeated until the tropopause temperature is reached.

The base level composition is for one mole of gas for which the $\mathrm{He} / \mathrm{H}_{2}$ ratio is 0.18 by number, and the other trace gases $\mathrm{H}_{2} \mathrm{O}, \mathrm{CH}_{4}, \mathrm{NH}_{3}, \mathrm{H}_{2} \mathrm{~S}$, and $\mathrm{Ar}$ are present in selected enrichments relative to solar. The $\mathrm{He} / \mathrm{H}_{2}$ ratio is in conformity with our previous Uranus studies and is based on the Voyager 2 IRIS determination for Uranus [Conrath et al., 1987]. It is also within error bounds of the solar $\mathrm{He} / \mathrm{H}_{2}$ ratio. From all studies $\mathrm{CH}_{4}$ was held at 30 times solar to reproduce approximately the observed $2 \%$ mixing ratio in the upper troposphere before the $\mathrm{CH}_{4}$ ice cloud begins to form. Studies in the IR indicate that Neptune and Uranus have similar temperatures at the 1 bar level [Orton et al., 1987], so we have chosen the temperature and pressure of the base level to reproduce the observed $101 \mathrm{~K}$ at 2.3 bars on Uranus by the Voyager RSS [Lindal et al., 1987].

\section{Radio Spectrum Model}

We used the radiative transfer code developed by de Pater and Massie [1985] to calculate the microwave brightness temperatures from the atmospheric model. The opacity in the millimeter part of Neptune's spectrum is influenced by both the collision-induced absorption of hydrogen as well as absorption of ammonia gas, while in the centimeter part of the spectrum it is controlled by ammonia gas, water vapor, and droplets.

\section{Data}

Recently de Pater and Richmond [1989] presented VLA observations of Neptune at $1.3,2,6$, and $20 \mathrm{~cm}$. Along with their observations they updated previously reported measurements to the same flux density scale and then converted the flux densities to brightness temperatures using the best known size for Neptune. The data are shown in Figure 1, with the VLA data points being solid circles and other observations shown as X's.

In our attempts to fit the observations we will concentrate on matching the VLA results, as recommended by de Pater and Richmond (1989). These data points have a higher accuracy than the other data. This may be due, in part, to confusion problems in single dish observations of a weak source. The VLA measurements also form a self consistent data set. Also note that the strong cluster of non VLA data points at $3 \mathrm{~cm}$, as well as the interferometric observations by Webster et al.( 1972) agree with our values.

\section{Fitting Attempts}

Along with the radio data in Figure 1, we present several attempts to fit the model spectrum. As shown before 
(e.g. de Pater and Massie, 1985) the spectrum for a solar composition atmosphere is too cold; this is indicated by the dotted line. The spectrum can be represented well with a constant low $\left(3 \times 10^{-6}\right) \mathrm{NH}_{3}$ mixing ratio throughout the atmosphere [de Pater and Richmond, 1989]; this curve is indicated by the dashed line. Note that this $\mathrm{NH}_{3}$ mixing ratio is $\sim 60$ times below the solar value. The solid lines in Figure 1 are spectra for Neptune if it were to have a composition like we suggested for Uranus (de Pater et al., 1989): $500 \times \mathrm{H}_{2} \mathrm{O}, 500 \times \mathrm{H}_{2} \mathrm{~S}$, and $15 \times \mathrm{NH}_{3}$. The upper solid line is a calculation for an atmosphere in thermochemical equilibrium; the lower solid line for an atmosphere with the same composition, but in which the $\mathrm{NH}_{3}$ mixing ratio is held constant once it reaches $3.0 \times 10^{-6}$ ( $\mathrm{T} \lesssim 245 \mathrm{~K}, \mathrm{P} \lesssim 35$ bars $)$, i.e. $\mathrm{NH}_{4} \mathrm{SH}$ is supersaturated. In a chemical equilibrium model, the formation of the $\mathrm{NH}_{4} \mathrm{SH}$ cloud will completely remove $\mathrm{NH}_{3}$ from the atmosphere if there is excess $\mathrm{H}_{2} \mathrm{~S}$ relative to $\mathrm{NH}_{3}$, since this reaction is equal molar in $\mathrm{H}_{2} \mathrm{~S}$ and $\mathrm{NH}_{3}$. This results in no $\mathrm{NH}_{3}$ in the upper atmosphere and brightness temperatures too high to match the data. The spectrum of radio data $(2-20 \mathrm{~cm})$ for Neptune can only be matched with a constant low $\mathrm{NH}_{3}$ mixing ratio of $3.0 \times 10^{-6}$ over the pressure region probed at these wavelengths (10 to 80 bars). So in all of our cloud model fits, once the $\mathrm{NH}_{3} \mathrm{mix}-$ ing ratio was decreased to $3.0 \times 10^{-6}$, it was held constant at this value. This implies either supersaturation in the $\mathrm{NH}_{4} \mathrm{SH}$ cloud (no $\mathrm{NH}_{4} \mathrm{SH}$ forms even though the equilibrium constant is exceeded), or rapid vertical transport that mixes $\mathrm{NH}_{3}$ rich gas below the $\mathrm{NH}_{4} \mathrm{SH}$ cloud with $\mathrm{NH}_{3}$ poor gas above. This requirement also occurred in modelling the Uranus spectrum but with $\mathrm{NH}_{3}$ at about $7 \times 10^{-7}$ for a global model [de Pater et al., 1989].

As mentioned above the $500 \times \mathrm{H}_{2} \mathrm{O}, 500 \times \mathrm{H}_{2} \mathrm{~S}$, and $15 \times \mathrm{NH}_{3}$ supersaturation model is typical of models that gave a good fit to the Uranus spectrum. However, the calculated brightness temperature at $20 \mathrm{~cm}$ falls well below the observed value of $318 \pm 16 \mathrm{~K}$. A decrease in the $\mathrm{NH}_{3}$ mixing ratio in the deep atmosphere to the solar value does not change the spectrum shortward of $6 \mathrm{~cm}$ and increases the $20 \mathrm{~cm}$ temperature only by $8 \mathrm{~K}$, still below the lower error bar.

While the $\mathrm{NH}_{3}$ mixing ratio is decreased considerably from that specified in Neptune's deep atmosphere by solution of $\mathrm{NH}_{3}$ into an aqueous ammonia cloud (up to $65 \%$ of the initial $\mathrm{NH}_{3}$ mixing ratio for $\mathrm{H}_{2} \mathrm{O}$ enrichments greater than about $100 \times$ solar), the aqueous ammonia cloud alone can not remove enough $\mathrm{NH}_{3}$ for even large water mixing ratios. In cases in which $\mathrm{H}_{2} \mathrm{O}$ is 500 times solar, the $\mathrm{NH}_{3}$ mixing ratio is $5 \times 10^{-4}$ at $\sim 300 \mathrm{~K}$. Since in our model $\mathrm{NH}_{3}$ is at $100 \%$ relative humidity, it can be interpreted that the cloud model would match the radio data if the relative humidity is on the order of $1 \%$. While in small areas this is possible, the radio data are global averages implying an unrealistically globally dry Neptune. Increasing the water enrichment to $1000 \times$ solar would only allow the global relative humidity to rise to $10 \%$.

Increasing the $\mathrm{H}_{2} \mathrm{~S}$ and/or $\mathrm{NH}_{3}$ mixing ratio will push the formation of the $\mathrm{NH}_{4} \mathrm{SH}$ cloud to deeper levels, thus decreasing the $\mathrm{NH}_{3}$ mixing ratio at higher temperatures and pressures, and increasing the brightness temperature of the planet at longer wavelengths. An increase in the $\mathrm{H}_{2} \mathrm{~S}$ mixing ratio from 500 to $2000 \times$ solar results in the $20 \mathrm{~cm}$ brightness temperature barely touching the lower error bounds, regardless of the ammonia mixing ratio ( 0.1 , 1 or $15 \times$ solar). A similar result can be obtained with
$\mathrm{H}_{2} \mathrm{~S}$ at $500 \times$ solar, and $\mathrm{NH}_{3}$ at 0.1 times solar. Hence, the lower error bounds at $20 \mathrm{~cm}$ can be matched if either the $\mathrm{H}_{2} \mathrm{~S}$ mixing ratio in the deep atmosphere is increased to $2000 \times$ solar, or the $\mathrm{NH}_{3}$ is decreased to $0.1 \times$ solar. At no time did the resultant curves go through the $20 \mathrm{~cm}$ data points, unless the $\mathrm{NH}_{3}$ mixing ratio was at least a factor of $\sim 60$ below the solar value.

The formation of $\mathrm{NH}_{4} \mathrm{SH}$ is incapable of reducing the $\mathrm{NH}_{3}$ to a mixing ratio of $3 \times 10^{-6}$ at $\sim 300 \mathrm{~K}$ for the following reason. The equilibrium constant, $\mathrm{Kp}$, for the formation of $\mathrm{NH}_{4} \mathrm{SH}$ is:

$$
\log _{10}\left(K_{p}\right)=14.83-\frac{4715}{T}
$$

at equilibrium this is equal to:

$$
\mathrm{K}_{\mathrm{p}}=\mathrm{P}^{2} \cdot \mathrm{NH}_{3} \cdot \mathrm{H}_{2} \mathrm{~S}
$$

where $\mathrm{P}$ is the atmospheric pressure in atmospheres and $\mathrm{NH}_{3}$ and $\mathrm{H}_{2} \mathrm{~S}$ are the volume mixing ratios of ammonia and hydrogen sulfide respectively. In our model atmospheres $\sim 300 \mathrm{~K}$ occurs at $\sim 80$ bars (the precise value depends upon the enrichment and whether or not a dry or wet adiabatic lapse rate was used). If at this level ammonia has been reduced to $3 \times 10^{-6}$ by $\mathrm{NH}_{4} \mathrm{SH}$ formation, then by using Equations 1 and 2 the $\mathrm{H}_{2} \mathrm{~S}$ mixing ratio must be 7: impossible. (We discuss the possibility of a non-adiabatic atmosphere in the next section).

Thus we are forced to assume that $\mathrm{NH}_{3}$ is subsolar throughout Neptune's atmosphere, unlike Uranus where a subsolar $\mathrm{NH}_{3}$ provided a poor fit and an above solar $\mathrm{NH}_{3}$ mixing ratio coupled with removal into clouds gave the best fits to the data. Our calculation show that the mixing ratio on Neptune should be $\sim 1 / 60 \times$ solar value.

\section{Discussion}

\section{Sub solar $N$}

One possible explanation is that Neptune is depleted in $\mathrm{NH}_{3}$ relative to Uranus. While a best fit to the Uranus microwave data at $20 \mathrm{~cm}$ requires a minimum mixing ratio of $10 \times$ solar $\mathrm{NH}_{3}$, a poor fit is possible with solar $\mathrm{NH}_{3}$. However the Neptune spectrum can only be matched at $20 \mathrm{~cm}$ by an $\mathrm{NH}_{3}$ mixing ratio $\sim 1 / 60$ times solar. This requires nearly a 100 fold gradient in $\mathrm{N}$ content in the primitive solar nebula from Uranus to Neptune. Since at present there is no compelling theory on the origin of these planets that supports this, we do not favor this explanation. We also note that the spectrum of Neptune can be fit at the other wavelengths by a composition similar to Uranus with only the $20 \mathrm{~cm}$ brightness temperature being a problem.

Another possibility is that $\mathrm{N}$ is present on both planets in the same mixing ratios but in the form of $\mathrm{N}_{2}$ on Neptune and $\mathrm{NH}_{3}$ on Uranus. While the dominant form of $\mathrm{N}$ in the outer solar system was probably $\mathrm{N}_{2}$, this was likely converted back to $\mathrm{NH}_{3}$ in the formation of the giant planets [Prinn and Fegley, 1981]. To photolyze $\mathrm{NH}_{3}$ back to $\mathrm{N}_{2}$ requires a warmer Neptune in the past enabling $\mathrm{NH}_{3}$ to get to the one bar level and above so it can absorb the solar UV before Rayleigh scattering blocks the penetration of solar UV. Also the interior of Neptune must be cold (a non-adiabatic atmosphere) and $\mathrm{H}_{2}$ poor so $\mathrm{N}_{2}$ is 
not converted back to $\mathrm{NH}_{3}$ by the Haber process. Scaling the $\mathrm{NH}_{3}$ photolysis loss rate from Jupiter $\left(4.0 \times 10^{-12}\right.$ grams cm${ }^{-2} \mathrm{sec}^{-1}$, (Atreya, et al., 1977) to Neptune gives a loss rate of $1.2 \times 10^{-13}$ grams cm-2 $\mathrm{sec}^{-1}$. Assuming Neptune's atmosphere to be at least 1 Earth mass, photolysis can convert only 0.2 of a solar mixing ratio of $\mathrm{NH}_{3}$ on Neptune over the age of the solar system. So we consider this unlikely. If $\mathrm{N}$ turns out to be in the form of $\mathrm{N}_{2}$ on Neptune then Voyager 2 will be able to detect it; $\mathrm{N}_{2}$ will not freeze out at Neptune's tropopause (saturation mixing ratio $-6.7 \times 10^{-2}$ ) and the Voyager 2 Ultraviolet Spectrometer can detect $\mathrm{N}_{2}$ as low as $10 \mathrm{ppb}$ at the microbar level.

\section{Non-adiabatic atmosphere}

In all our calculations we assumed that the thermal structure was adiabatic. This was important in showing that $\mathrm{NH}_{4} \mathrm{SH}$ formation is incapable of reducing the $\mathrm{NH}_{3}$ mixing ratio to $3 \times 10^{-6}$ at $300 \mathrm{~K}$. But we can ask the question: what is the pressure for a given temperature $(300 \mathrm{~K})$, $\mathrm{H}_{2} \mathrm{~S}$ mixing ratio $\left(10^{-2}, \approx 500 \times\right.$ solar $)$ and $\mathrm{NH}_{3}$ mixing ratio $\left(3 \times 10^{-6}\right)$. From Equations 1 and 2, the pressure must be 2000 bars. In an adiabatic atmosphere, the temperature at this pressure level is $600 \mathrm{~K}$, twice $300 \mathrm{~K}$. Thus Neptune's atmosphere would have to be more isothermal than an adiabatic one with a lapse rate nearly half the adiabat. However, Neptune emits more energy than it receives from the Sun, and it is likely that in the deep atmosphere the heat transport is done by convection. This implies an adiabatic thermal structure, so we consider this alternate thermal structure unlikely.

\section{Synchrotron radiation}

De Pater and Goertz [1989] have proposed that Uranus and Neptune do have similar compositions and that the difference between the two $20 \mathrm{~cm}$ brightness temperatures is that Neptune's is a combination of thermal and synchrotron emission while Uranus' is thermal alone. This seems to be the likeliest explanation. The electron population and magnetic field required for this interpretation will be directly verifiable by Voyager 2 experiments.

Acknowledgements. P. N. Romani acknowledges support at NASA Goddard Space Flight Center, Greenbelt, Maryland, from NASA contract NAS5-30134, I de Pater from NSF grant AST-8405358 and AST-8900156 to the University of California in Berkeley and the Alfred P. Sloan Foundation, S. K. Atreya from NSG-7404 and NASA grant NAGW-1771.

\section{References}

Atreya, S. K., T. M. Donahue, and W. R. Kuhn 1977, The distribution of ammonia and its photochemical products on Jupiter, Icarus, 31, 348-355.

Conrath, B., D. Gautier, R. Hannel, G. Lindal, and A. Marten, The helium abundance of Uranus from Voyager measurements, J. Geophys. Res., 92, 15003$15010,1987$.

Conrath, B. J., J. C. Pearl, J. F. Appleby, G. F. Lindal, G. S. Orton, and B. Bezard, Thermal structure and heat balance of Uranus, paper presented at Uranus colloquium, Pasadena, June 28 to July 1, 1988

de Pater, I., and S. T. Massie, Models of the millimetercentimeter spectra of the giant planets, Icarus, 62, 143-171, 1985.

de Pater, I., and C. K. Goertz, Synchrotron radiation from Neptune: Neptune's magnetic field and electron population, Geophys. Res. Let., 16, 97-100, 1989.

de Pater, I., and M. Richmond, Neptune's microwave spectrum from $1 \mathrm{~mm}$ to $20 \mathrm{~cm}$, Icarus, In press, 1989 .

de Pater, I., P. N. Romani, and S. K. Atreya, Uranus deep atmosphere revealed, Icarus, In press 1989.

Lindal, G. F., J. R. Lyons, D. E. Sweetnam, V. R. Eshleman, D. P. Hinson, and G. L. Tyler, The atmosphere of Uranus: results of radio occultation measurements with Voyager 2, J. Geophys. Res., 92, 14987-15001, 1987.

Orton, G. S., D. K. Aitken, C. Smith, P. F. Rouche, J. Caldwell, R. Snyder, The spectra of Uranus and Neptune at 8-14 and 17-23 $\mu \mathrm{m}$, Icarus, 70, 1-12, 1987.

Prinn, R. G, and B. Fegley, Jr., Kinetic inhibition of CO and $\mathrm{N}_{2}$ reduction in circumplanetary nebulae: implications for satellite composition, Astrophys. J., 249, 308-317, 1981.

Webster, W. HJ. Jr., A. C. Webster, and G. T. Webster, 1972, Interferometric observations of Uranus, Neptune, and Pluto at wavelengths of 11.1 and 3.7 centimeters, Astrophys. J., 174, 679-684.

Atreya, Sushil K., Department of Atmospheric, Oceanic and Space Sciences, The University of Michigan, Ann Arbor, MI 48109-2143.

de Pater, Imke, Astronomy Department, Campbell Hall 601, University of California, Berkeley, CA 94720.

Romani, Paul N., Code 693.2, NASA/Goddard Space Flight Center, Greenbelt MD 20771.

(Received May 22, 1989;

revised: June 26, 1989;

accepted: June 26, 1989.) 